\title{
Bifurcation and Chaos Analysis of Gear Pair System Based on Crack Rotor-Bearing System with Rub-Impact Effect
}

\author{
Cai-Wan Chang-Jian ${ }^{1, *}$, Cheng-Chi Wang ${ }^{2}$ and Li-Ming Chu ${ }^{3}$ \\ 1 I-Shou University; cwchangjian@isu.edu.tw \\ 2 National Chin-Yi University of Technology; wcc@ncut.edu.tw \\ National Taitung University; lmchu@nttu.edu.tw \\ * Correspondence: cwchangjian@isu.edu.tw; Tel.: +886-7-6577711 ext.3231 \\ Academic Editor: name \\ Received: date; Accepted: date; Published: date
}

\begin{abstract}
This study performs a systematic analysis of the dynamic behavior of a crack rotor-bearing system with rub-impact effect. The dynamic orbits of the system are observed using bifurcation diagrams plotted using the dimensionless damping coefficient, the dimensionless unbalance parameter and the dimensionless rotational speed ratio as control parameters. The analysis methods employed in this study include the dynamic trajectories of the crack rotor-bearing system, power spectra, Poincaré maps and bifurcation diagrams. Lyapunov exponent and fractal dimensional analysis are also used to identify the onset of chaotic motion. The numerical results reveal that the system exhibits a diverse range of periodic, sub-harmonic, quasi-periodic and chaotic behaviors. The results presented in this study provide an understanding of the operating conditions under which undesirable dynamic motion takes place in a crack rotor-bearing system and therefore serve as a useful source of reference for engineers in designing and controlling such systems.
\end{abstract}

Keywords: rotor-bearing system; cracked rotor; rub-impact

PACS: J0101

\section{Introduction}

Cracked rotor and rub-impact between rotor/stator are both common faults found in turbo-machineries. Those faults will induce some possible fatigue. The diagnosis the cracked rotor or rub-impact effect can help researchers or engineers understand how to escape the undesired vibration and fatigues. Most literatures also emphasize the importance of cracked rotor or rub-impact effect. Sabnavis et al. [1] presented a very comprehensive study on vibration of cracked shaft and found some interesting vibration phenomena induced by rotor crack. Jun et al. [2] found that cracked rotor would cause periodic steady state lateral vibrations at twice and thrice the rotation speed due to breathing of the crack under gravity loading and thus proved that the relations between dynamic behaviors of system and cracked rotor. Zhou et al. [3] took some experiments to prove cracked rotor would produce some problems of dynamical systems and thus evidenced numerical results of formal literatures. Darpe et al. [4-5] investigated the dynamic behaviors of two cracked rotors and also discussed the effect of bow. They found the dynamics of the system would become more seriously with effects of cracked rotor and bow coexisting.

\section{Mathematical Modeling}

Figure 1 shows a flexible crack rotor supported by two oil film journal bearings on a parallel with nonlinear springs suspension under rub-impact between rotor and stator. $\mathrm{O}_{\mathrm{m}}$ is the center of rotor gravity, $\mathrm{O}_{1}$ is the geometric center of the bearing, $\mathrm{O}_{2}$ is the geometric center of the rotor, $\mathrm{O}_{3}$ is 
the geometric center of the journal. From the equilibrium of force, the forces applied to the journal center $\mathrm{O}_{3}$ are

$$
\begin{aligned}
& F_{x}=f_{e} \cos \varphi+f_{\varphi} \sin \varphi=K_{s}\left(1-\frac{\delta_{1} \delta_{2}}{2}\right)\left(X_{2}-X_{3}\right) / 2 \\
& F_{y}=f_{e} \sin \varphi-f_{\varphi} \cos \varphi=K_{s}\left(1-\frac{\delta_{1} \delta_{2}}{2}\right)\left(Y_{2}-Y_{3}\right) / 2
\end{aligned}
$$

where $\quad \delta_{1}=\frac{1}{2} L_{R} \frac{\bar{\lambda}_{\xi}-\bar{\lambda}_{\eta}}{\left(L_{R}+\bar{\lambda}_{\xi}\right)\left(L_{R}+\bar{\lambda}_{\eta}\right)} \quad, \quad \delta_{2}=\frac{1}{\delta_{1}}-\frac{\bar{\lambda}_{\xi}+\bar{\lambda}_{\eta}}{\bar{\lambda}_{\xi}-\bar{\lambda}_{\eta}}-\frac{2 L_{R}}{\bar{\lambda}_{\xi}-\bar{\lambda}_{\eta}} \quad, \quad L_{R}=\frac{L_{1}}{3 R\left(1-v^{2}\right)} \quad$ ， $\bar{\lambda}_{\xi}=\frac{\pi R^{3} E}{\left(1-v^{2}\right)} c_{\xi} \int_{-\frac{b}{R}}^{\frac{b}{R}} \int_{0}^{\frac{\eta}{R}} 32\left[1-\left(\frac{\xi}{R}\right)^{2}\right]\left(\frac{\bar{\eta}}{R}\right) F_{2}^{2}\left(\frac{\bar{\eta}}{R}\right) d\left(\frac{\bar{\eta}}{R}\right) d\left(\frac{\xi}{R}\right) ， \quad \bar{\lambda}_{\eta}=\frac{\pi R^{3} E}{\left(1-v^{2}\right)} c_{\eta} \int_{0}^{\frac{b}{R}} \int_{0}^{\frac{\eta}{R}} 32\left(\frac{\xi}{R}\right)^{2}\left(\frac{\bar{\eta}}{R}\right) F_{1}^{2}\left(\frac{\bar{\eta}}{R}\right) d\left(\frac{\bar{\eta}}{R}\right) d\left(\frac{\xi}{R}\right)$ ， $h=2 \sqrt{R^{2}-\xi^{2}}, \eta(\xi)=a-R+\sqrt{R^{2}-\xi^{2}}$ is the local depth of crack, $L_{1}$ is the length of shaft between both supported ends, $R$ is the radius of journal, $E$ is the Young's Modulus, $a$ is the depth of crack and $v$ is the poisson ratio. The following parameters $F_{1}\left(\frac{\eta}{h}\right)$ and $F_{2}\left(\frac{\eta}{h}\right)$ will be defined, then.

$$
\begin{aligned}
& F_{1}\left(\frac{\eta}{h}\right)=\sqrt{\frac{2 h}{\pi \eta \tan \left(\frac{\pi \eta}{2 h}\right)}}\left\{0.923+0.199[1-\sin (\pi \eta)]^{4}\right\} / \cos \left(\frac{\pi \eta}{2 h}\right) \\
& F_{2}\left(\frac{\eta}{h}\right)=\sqrt{\frac{2 h}{\pi \eta \tan \left(\frac{\pi \eta}{2 h}\right)}}\left\{0.752+2.02\left(\frac{\eta}{h}\right)+0.37[1-\sin (\pi \eta)]^{3}\right\} / \cos \left(\frac{\pi \eta}{2 h}\right)
\end{aligned}
$$

From assumptions (a) to (c), it means that the whirling is considered, while the wobbling is neglected. The equations of motion of $\mathrm{O} 2$ in the Cartesian coordinates and the equations of motion of the bearing center could be written as

$$
m+C_{2} X_{2}^{\&}+K_{s}\left(1-\frac{\delta_{1} \delta_{2}}{2}\right)\left(X_{2}-X_{3}\right)+\frac{\delta_{1} f}{2} K_{s}\left[\left(X_{2}-X_{3}\right) \cos (2 \omega t)+\left(Y_{2}-Y_{3}\right) \sin (2 \omega t)\right]=m \rho \omega^{2} \cos \phi+R_{x}
$$

(3)

$$
m \frac{\delta_{2}}{2}+C_{2} \Psi_{2}^{\&}+K_{s}\left(1-\frac{\delta_{1} \delta_{2}}{2}\right)\left(Y_{2}-Y_{3}\right)+\frac{\delta_{1} f}{2} K_{s}\left[\left(X_{2}-X_{3}\right) \sin (2 \omega t)-\left(Y_{2}-Y_{3}\right) \cos (2 \omega t)=m \rho \omega^{2} \sin \phi-m g+R_{y}\right.
$$

(4)

$$
\begin{aligned}
& m_{0} C_{1} X_{1}^{\&}+K_{1} X_{1}+K_{2} X_{1}^{3}=F_{x} \\
& m_{0} C_{1}{ }_{1} K_{1} Y_{1}+K_{2} Y_{1}^{3}=-m_{0} g+F_{y}
\end{aligned}
$$

where $g$ is the acceleration of gravity, $F_{x}$ and $F_{y}$ are the components of the oil film force. $R_{x}$ and $R_{y}$ are the rub-impact forces in the horizontal and vertical directions.

Equations (3)-(6) describe a nonlinear dynamic system. The approximate solution of these coupled nonlinear differential equations can be obtained by numerical methods.

\section{Results}

The nonlinear dynamic equations presented in equations (3) to (6) for the crack rotor-bearing system were solved using the fourth order Runge-Kutta method. The time step in the iterative solution procedure was assigned a value of $\pi / 300$ and the termination criterion was specified as an error tolerance of less than 0.0001 . The time series data corresponding to the first 800 revolutions of the rotor-bearing system were deliberately excluded from the dynamic analysis to ensure that the analyzed data related to steady-state conditions. The sampled data were used to generate the dynamic trajectories, Poincaré maps and bifurcation diagrams of the rotor-bearing system in order to obtain a basic understanding of its dynamic behavior. The maximum Lyapunov exponent and the fractal dimension measure were then used to identify the onset of chaotic motion. 
Figure 2 presents the bifurcation diagram for the dimensionless displacement of the rotor center and bearing center in the horizontal direction using the dimensionless rotating speed ratio $s$ as a bifurcation parameter. Note that the rotor-bearing system is assumed to be without rub-impact effect in this case. It can be observed that both the rotor center and bearing center exhibit periodic or nT-periodic motions at low values of the dimensionless rotating speed ratio, i.e. $s \leq 2.80$. Besides, we also find that the so-called "Jump phenomena" is also happening at $s=1.20$ for rotor center. However, as $s$ is increased from 2.80 to 3.40 , the nT-periodic motion is replaced by non-periodic motion both for rotor and bearing dynamic trajectories. As the dimensionless rotating speed ratio is further increased from $s=3.40$ to $s=5.20$, the non-periodic motion transits to periodic motion and then the dynamic trajectory becomes sub-harmonic motion of $8 T$-period as $s>5.20$. For $7.80<s<8.90$, the trajectory performs non-periodic motion and it becomes synchronous 1T-periodic motion as $s>8.90$, finally. Figure 3 are the history of Poincaré maps of rotor dynamic behaviors with rub-impact effect at $s=0.10,0.20,0.50,1.00,2.00,2.30,2.40,2.60,2.80,3.00,3.20,3.60,4.00,6.00$ and 8.00. We can find that the dynamic trajectory history performs $1 \mathrm{~T} \Rightarrow \mathrm{nT} \Rightarrow$ quasi-peirodic motions. Figure 4 is the bifurcation diagram of rotor dynamic behaviors in the horizontal direction with rub-impact at $s=0.50$ using dimensionless damping coefficient, $\xi_{1}$ as bifurcation parameter. The dynamic trajectories of the rotor center perform non-periodic at low values of the dimensionless damping coefficient, i.e. $\xi_{1}<0.03$ and then 3T-period and period motion would be found at $0.03 \leq \xi_{1} \leq 0.032$ and $0.032 \leq \xi_{1} \leq 0.034$ respectively. As the dimensionless damping coefficient is further increased from $\xi_{1}=0.034$ to $\xi_{1}=0.05$, the non-periodic motion happen and non-periodic motions transits to sub-harmonic motion of 2T-periodic motions as $\xi_{1}>0.05$, but non-periodic dynamic response would occur again at $0.056 \leq \xi_{1} \leq 0.064$. Finally, the periodic motion perform at higher values of dimensionless damping coefficient $\xi_{1}>0.064$. Figure 5 are the Poincaré maps with rub-impact effect at $\xi_{1}=0.0001,0.001,0.01,0.02,0.028,0.03,0.032$ and 0.04 . The simulation results show that quasi-periodic motions are found at $\xi_{1}=0.0001$ and 0.001 , chaotic motions are found at $\xi_{1}=0.01$, $0.02,0.028$ and $0.04,3 T$-periodic motion is found at $\xi_{1}=0.03$ and periodic motion is found at $\xi_{1}=$ 0.032 .

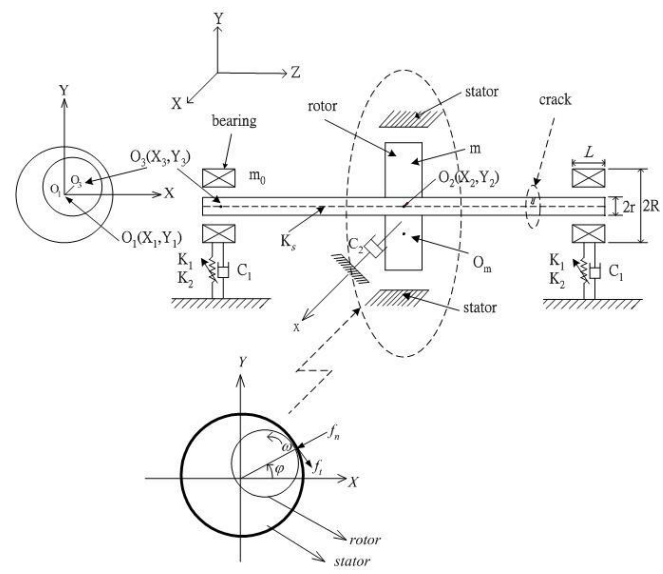

Fig.1 Dynamic model of rotor-bearing system with crack effect and rub-impact effect.
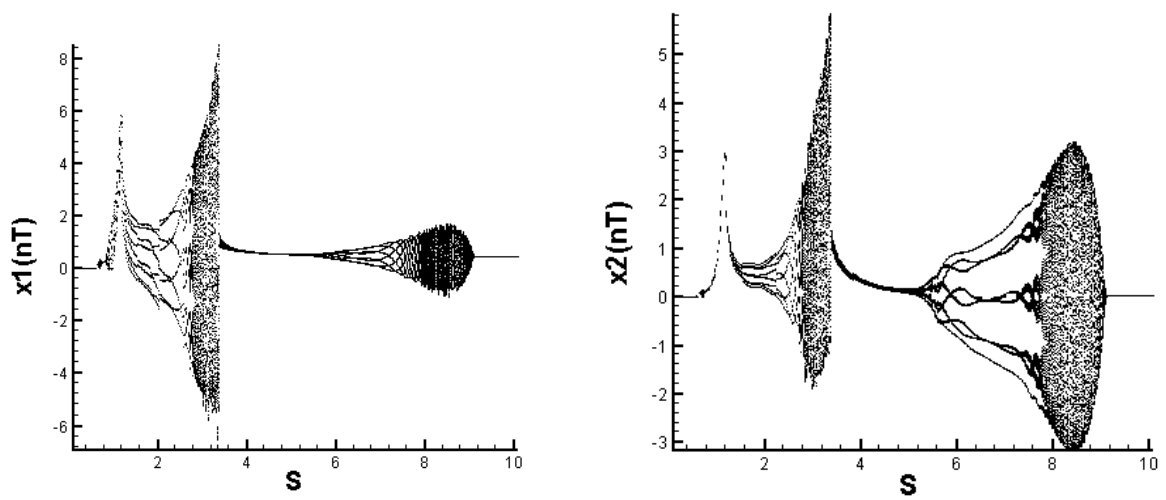
Fig.2 Bifurcation diagram of rotor and bearing dynamic behaviors with rub-impact effect using dimensionless rotating speed ratio, $s$, as bifurcation parameter.
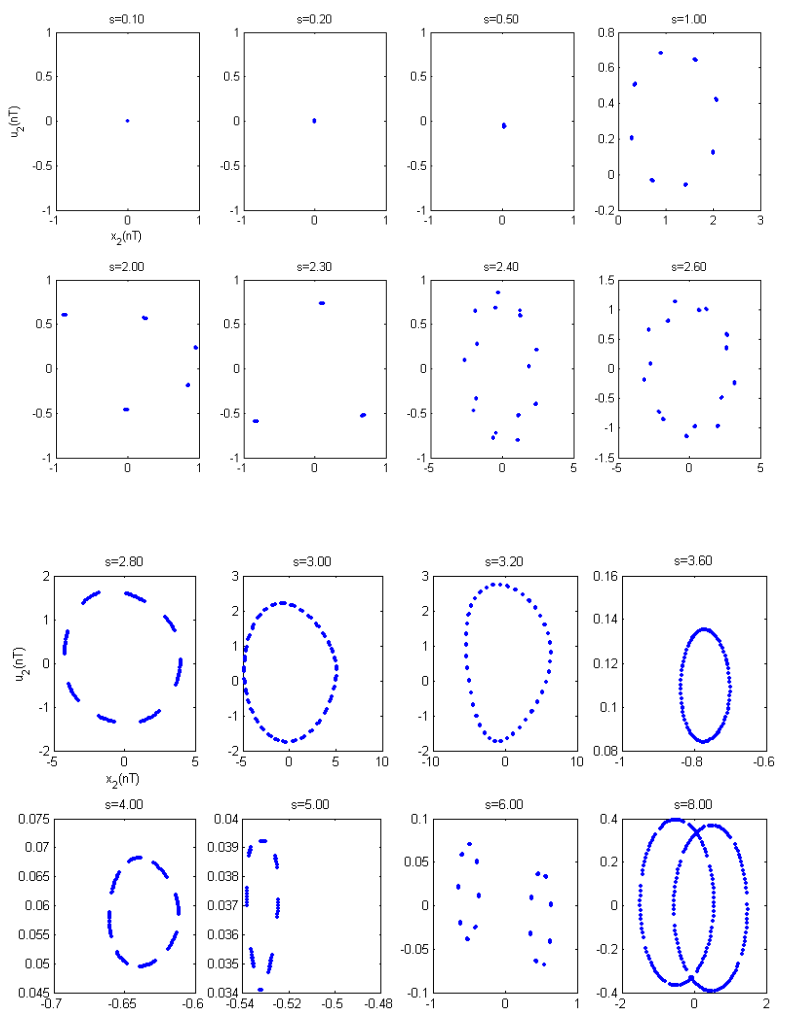

Fig.3 The history of Poincaré maps of rotor dynamic behaviors with rub-impact effect at $s=0.10,0.20$, $0.50,1.00,2.00,2.30,2.40,2.60,2.80,3.00,3.20,3.60,4.00,6.00$ and 8.00 .

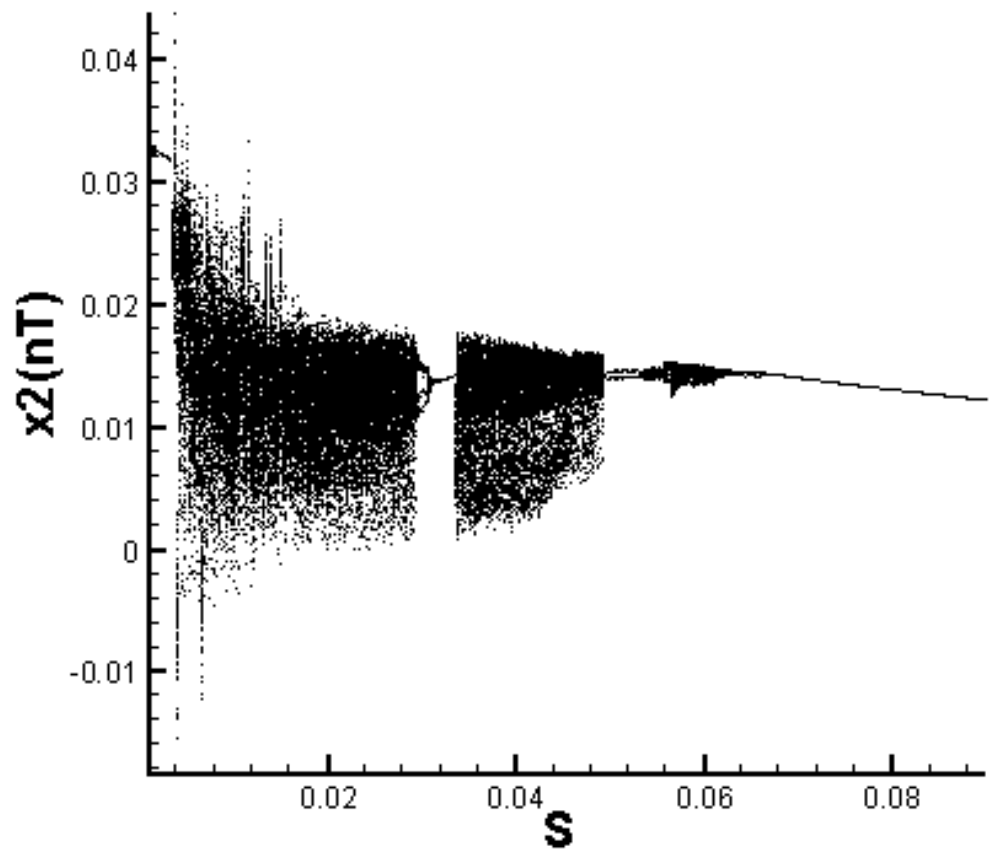


Fig.4 Bifurcation diagram of rotor dynamic behaviors in horizontal direction with rub-impact at $s=0.5$ effect using dimensionless damping coefficient, $\xi_{1}$, as bifurcation parameter.
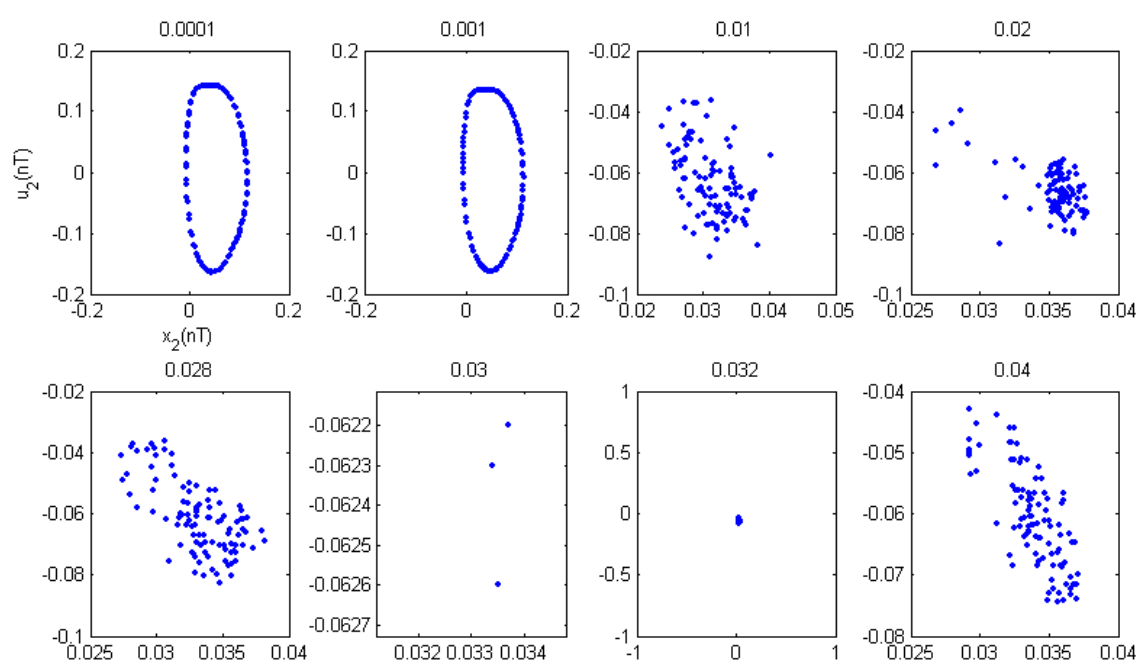

Fig.5 The history of Poincaré maps of rotor dynamic behaviors with rub-impact effect at $\xi_{1}=0.0001$,

$$
0.001,0.01,0.02,0.028,0.03,0.032 \text { and } 0.04 \text {. }
$$

Acknowledgments: Financial support for this work was provided by the Ministry of Science and Technology Taiwan, under the contract MOST 106-2221-E-214-023.

\section{References}

1. Sabnavis G.; Kirk R.G.; Kasarada M.; Quinn D. Cracked shaft detection and diagnostics: a literature review. Shock Vibr Digest 2004; Volume 36, pp. 287-296.

2. Jun O.S.; Earmme H.Y.; Lee C.W. Modelling and vibration analysis of a simple rotor with breathing crack. J Sound Vibr 1992; Volume 155, pp. 273-90.

3. Zhou T.; Xu Z.; Han W. Experimental analysis of a cracked rotor. J. Dyna Sys Meas Control 2005; Volume 127, pp. 313-20.

4. Darpe A.K.; Gupta K.; Chawla A. Dynamics of a two-cracked rotor. J Sound Vibr 2003; Volume 259, pp. 649-75.

5. Darpe A.K.; Gupta K.; Chawla A. Dynamics of a bowed rotor with a transverse surface crack. J Sound Vibr 2006; Volume 296, pp. 888-907. 